\title{
Studies on the Occurrence, Cytology, Fertility and Breeding Behaviour of Aneuploids in Induced Autotetraploid Job's Tears
}

\author{
Panuganti N. Rao \\ Department of Botany, Andhra University, Waltair, India
}

Received August 20, 1974

The occurrence and frequency of aneuploids in relation to their effects on fertility and stability of autotetraploids have been studied in many plant species (see Ellerström and Sjödin 1966, Ahloowalia 1971). In the present investigation, the occurrence and meiotic behaviour of aneuploids, their fertility and breeding behaviour in induced autotetraploid $(4 \mathrm{n}=40)$ Job's tears (Coix lacryma-jobi L.), were studied and the results obtained are presented below.

\section{Materials and methods}

Cytological investigations were restricted to studies of pollen mother cells from acetocarmine squash preparations.

Pollen sterility was determined by staining with acetocarmine. Those unstained or imperfectly stained were scored as sterile. In each case, a minjmum of 1,000 grains coming from three different male spikelets were counted.

For female fertility, all the mature false fruits in a plant on a given day, towards the end of the crop season, were harvested; hard and brownish-black false fruits were scored as fertile, the soft and chalky-white ones as sterile.

\section{Results and discussion}

\section{Occurrence of aneuploids}

A large progeny was raised from an open pollinated seed lot collected from three colchicine induced autotetraploid plants. Of these, 70 plants were scored cytologically for distribution of chromosome numbers. One of the plants showed $2 n=21$ which obviously was resultant of apomictic development of an unfertilized egg or some other nucleus of the embryo sac containing an extra chromosome $(2 n+1)$ due to irregular separation during anaphase $I$ in the parent (Venkateswarlu and Rao 1974). The cytological findings of the rest of the 69 plants, together with the theoretically possible gametic unions and zygotic chromosome numbers, and their expected frequencies are given in Table 1. Theoretical expectations of the gametic combinations were arrived at on the basis of an analysis of the frequencies of cells with various anaphase I distributions of chromosomes in the parental plant (Rao, unpublished). In this calculation, cells containing laggards were ignored. Of the 134 cells, free from laggards, observed at anaphase I, 92 (about $69 \%$ ) showed 20: 20, 32 (about $24 \%$ ) and 10 (about 7\%) cells respectively showed 19:21 and 
Table 1. Theoretically possible gametic combinations and zygotic chromosome numbers with their expected and observed frequencies in the progenies

of autotetraploid $(4 n=40)$ Job's tears

\begin{tabular}{|c|c|c|c|c|c|}
\hline \multirow{2}{*}{$\begin{array}{c}\text { Gametic } \\
\text { combinations* }\end{array}$} & \multirow{2}{*}{$\begin{array}{l}\text { Chromosome } \\
\text { numbers }(4 n) \\
\text { in the progeny }\end{array}$} & \multicolumn{2}{|c|}{ Expected } & \multicolumn{2}{|c|}{ Observed } \\
\hline & & Frequency & $\%$ & Frequency & $\%$ \\
\hline $18 \times 18$ & $36^{* *}$ & 1 & 0.15 & - & - \\
\hline $18 \times 19$ & $37 * *$ & 6 & 0.89 & - & - \\
\hline $19 \times 19$ & $38^{* *}$ & 9 & 1.33 & - & - \\
\hline $20 \times 18$ & $38^{* *}$ & 36 & 5.33 & - & - \\
\hline $19 \times 20$ & 39 & 108 & 15.98 & 5 & 7.25 \\
\hline $18 \times 21$ & $39^{* *}$ & 6 & 0.89 & - & - \\
\hline $20 \times 20$ & 40 & 324 & 47.93 & 56 & 81.16 \\
\hline $19 \times 21$ & $40^{* *}$ & 18 & 2.66 & - & - \\
\hline $18 \times 22$ & $40^{* *}$ & 2 & 0.30 & 一 & - \\
\hline $21 \times 20$ & 41 & 108 & 15.98 & 8 & 11.59 \\
\hline $19 \times 22$ & $41^{* *}$ & 6 & 0.89 & - & - \\
\hline $20 \times 22$ & $42^{* *}$ & 36 & 5.33 & - & - \\
\hline $21 \times 21$ & $42^{* *}$ & 9 & 1.33 & - & - \\
\hline $21 \times 22$ & $43^{* *}$ & 6 & 0.89 & - & - \\
\hline $22 \times 22$ & $44^{* *}$ & 1 & 0.15 & - & - \\
\hline
\end{tabular}

* Calculated on the basis of anaphase I separation of chromosomes in the autotetraploid (Rao, unpublished).

** Zygotes formed by gametes having more than one chromosome difference.

Table 2. Chromosome associations at diakinesis and metaphase I in the 39-chromosome plant of Job's tears

\begin{tabular}{|c|c|c|c|c|c|}
\hline \multicolumn{4}{|c|}{ Chromosome associations } & \multirow{2}{*}{$\begin{array}{l}\text { No. of } \\
\text { cells }\end{array}$} & \multirow{2}{*}{$\%$} \\
\hline IV & III & II & I & & \\
\hline 9 & 1 & - & - & 1 & 1.32 \\
\hline 9 & - & 1 & 1 & 1 & 1.32 \\
\hline 8 & 1 & 2 & - & 2 & 2.63 \\
\hline 8 & - & 3 & 1 & 2 & 2.63 \\
\hline 7 & 1 & 4 & - & 5 & 6.58 \\
\hline 7 & - & 5 & 1 & 3 & 3.95 \\
\hline 6 & 1 & 6 & - & 7 & 9.21 \\
\hline 6 & - & 7 & 1 & 6 & 7.89 \\
\hline 5 & 1 & 8 & - & 10 & 13.16 \\
\hline 5 & - & 9 & 1 & 7 & 9.21 \\
\hline 4 & 1 & 10 & - & 9 & 11.84 \\
\hline 4 & - & 11 & 1 & 8 & 10.53 \\
\hline 3 & 1 & 12 & - & 4 & 5.26 \\
\hline 3 & - & 13 & 1 & 4 & 5.26 \\
\hline 2 & 1 & 14 & - & 4 & 5.26 \\
\hline 2 & - & 15 & 1 & 2 & 2.63 \\
\hline \multirow[t]{2}{*}{1} & 1 & 16 & - & 1 & 1.32 \\
\hline & & & & Total & \\
\hline
\end{tabular}


18:22 separation of chromosomes. Thus the different distributions of 20:20, 19:21 and 18:22 were approximately in the proportion of $9: 3: 1$ respectively. Assuming i) occurrence of similar distributions with the same relative frequencies in female gametogenesis, ii) all gametes, euploid and aneuploid, derived from such distributions are functional and iii) random mating of gametes, the expected zygotic contents of chromosomes and their frequencies were calculated. However, as is evident from Table 1, none of the zygotic chromosomal possibilities except 39,40 and 41 were actually realized. This might possibly suggest that gametes having 18 or 22 chromosomes were not functional. This does not, however, seem to be the case because in both natural and artificial interspecific crosses between $C$. aquatica

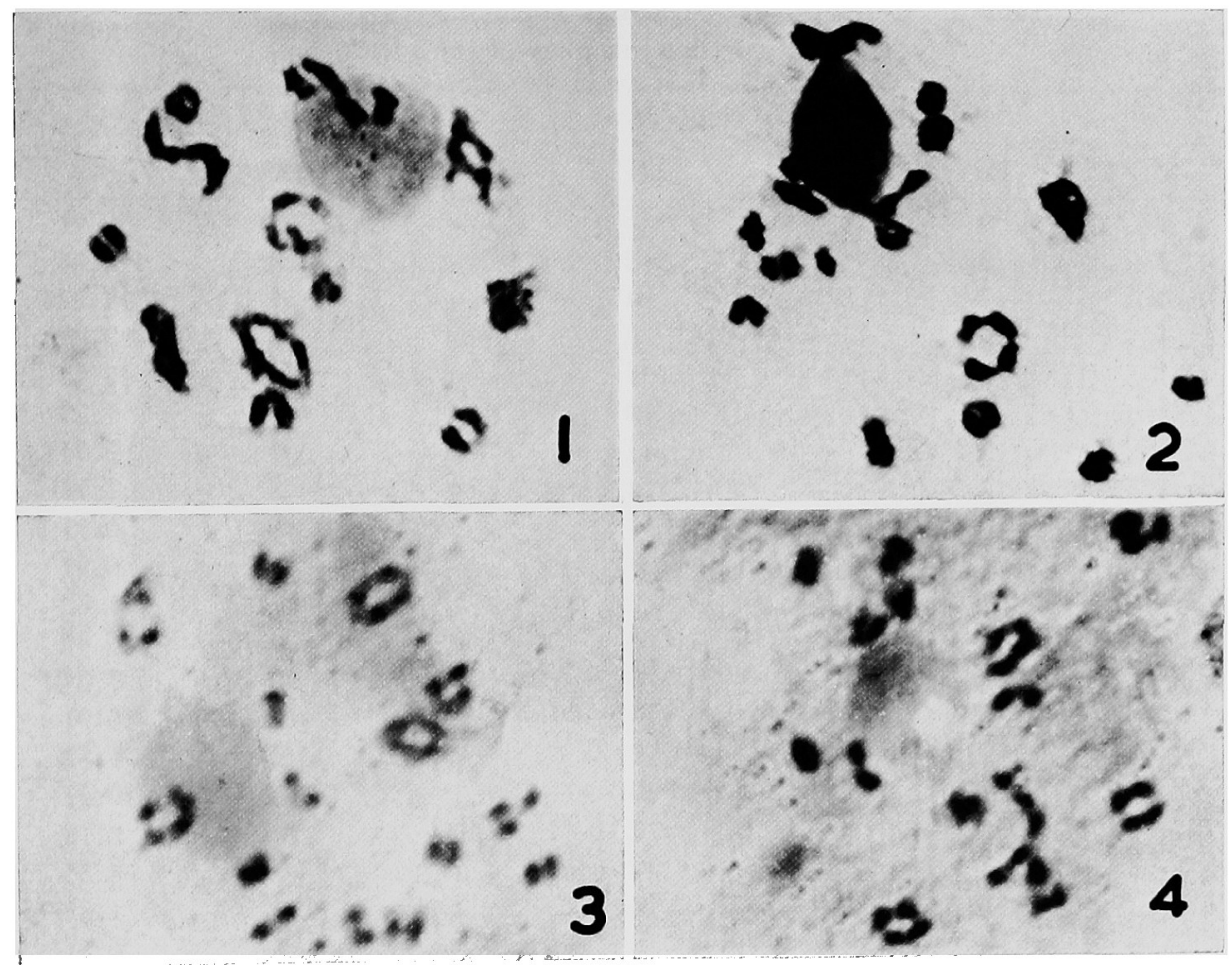

Figs. 1-4. 1 and 2. Diakinesis in 39-chromosome plant. $\times 1050 . \quad 1,7$ IV, 1 III (middle of the cell) and 4 II. 2, 2 IV, 15 II and 1 I. 3 and 4 . Diakinesis in 41-chromosome plant. 3, 5 IV, $1 \mathrm{III}$ and 9 II. 4, 1 V, 4 IV and $10 \mathrm{II}$.

Roxb. $(2 \mathrm{n}=10)$ and tetraploid C. lacryma-jobi $(4 \mathrm{n}=40)$, made reciprocally, $\mathrm{F}_{1}$ hybrids with chromosome numbers of $2 \mathrm{n}=24,25,26$ and 27 (with 19, 20, 21 and 22 chromosomes of lacryma-jobi respectively and 5 of aquatica) occurred indicating that aneuploid gametes even with 22 chromosomes, in the tetraploid parent, functioned successfully in fertilization even on the male side (Rao unpublished). This being so, then it might be argued that the progeny examined is too small to enable recovery of the remaining zygotic classes. But a subsequent cytological study of 108 tetraploid plants, made on different occasions for screening interspecific hybrids, also did not reveal the presence of plants with less than 39 and more than 41 chromo- 
somes. Among these 108 plants, $88(81.48 \%)$ showed $4 n=40,13(12.04 \%)$ had $4 n=41$ and $7(6.48 \%)$ showed $4 n=39$ chromosomes. These were not included in the data given in Table 1, because the exact chromosome numbers of the parents from which these 108 plants came were not known, and the observed progenies in Table 1 have all come from plants having $4 n=40$. Eighty six plants of a selfed progeny of the tetraploid, examined cytologically, also did not show chromosome numbers beyond the 39-41 range. Furthermore, none of the progenies of aneuploid plants $(4 n=39$ and 41$)$ studied contained any plant outside the 39-41 chromosome range (Table 5). It is, therefore, quite possible that the remaining zygotic classes were not recovered not because of the small population examined or non-

Table 3. Chromosome associations at diakinesis and metaphase I in the 41-chromosome plant of Job's tears

\begin{tabular}{|c|c|c|c|c|c|c|}
\hline \multicolumn{5}{|c|}{ Chromosome associations } & \multirow{2}{*}{$\begin{array}{l}\text { No. of } \\
\text { cells }\end{array}$} & \multirow{2}{*}{$\%$} \\
\hline V & IV & III & II & I & & \\
\hline 1 & 9 & - & - & - & 1 & 0.91 \\
\hline 1 & 8 & - & 2 & - & 4 & 3.64 \\
\hline 1 & 7 & - & 4 & - & 8 & 7.27 \\
\hline 1 & 6 & - & 6 & - & 8 & 7.27 \\
\hline 1 & 5 & - & 8 & - & 14 & 12.73 \\
\hline 1 & 4 & - & 10 & - & 8 & 7.27 \\
\hline 1 & 3 & - & 12 & - & 3 & 2.73 \\
\hline 1 & 2 & - & 14 & - & 3 & 2.73 \\
\hline- & 10 & - & - & 1 & 1 & 0.91 \\
\hline- & 9 & 1 & 1 & - & 1 & 0.91 \\
\hline- & 9 & - & 2 & 1 & 2 & 1.82 \\
\hline- & 8 & - & 4 & 1 & 6 & 5.45 \\
\hline- & 7 & - & 6 & 1 & 8 & 7.27 \\
\hline- & 6 & - & 8 & 1 & 12 & 10.91 \\
\hline- & 5 & 1 & 9 & - & 2 & 1.82 \\
\hline- & 5 & - & 10 & 1 & 12 & 10.91 \\
\hline- & 4 & - & 12 & 1 & 9 & 8.18 \\
\hline- & 3 & - & 14 & 1 & 5 & 4.55 \\
\hline- & 2 & - & 16 & 1 & 2 & 1.82 \\
\hline \multirow[t]{2}{*}{-} & 1 & - & 18 & 1 & 1 & 0.91 \\
\hline & & & & & 110 & \\
\hline
\end{tabular}

functional nature of the 18- or 22-chromosome aneuploid gametes, but that zygotes outside the 39-41 chromosome range are inviable. Thus the limits of aneuploidy in the autotetraploid were set most probably by the embryo abortion.

There were no morphological differences between aneuploids and euploids and the two can be distinguished only by a count of chromosome numbers.

Cytology and origin of the 39- and 41-chromosome plants

Chromosome pairing was studied at diakinesis and metaphase $\mathrm{I}$ in aneuploids. Although the data on chromosome associations were recorded from only one of the plants of each of the aneuploid classes in $C_{1}$ generation, all the aneuploid plants obtained in $\mathrm{C}_{1}$ and $\mathrm{C}_{2}$ generations were, nevertheless, critically examined at these 


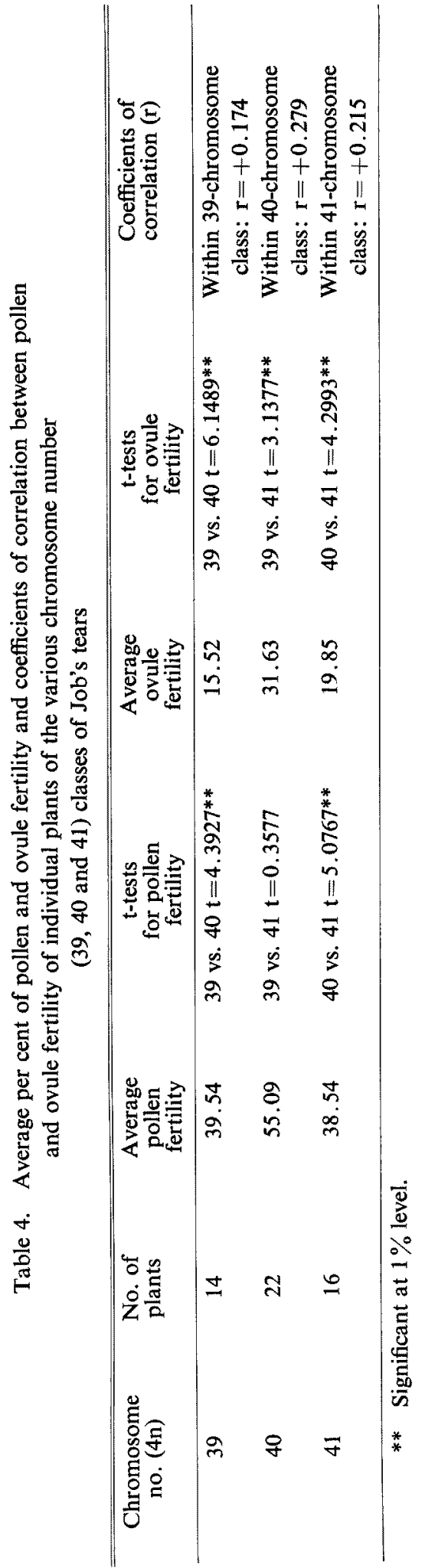


stages for chromosomal associations for an understanding of the origin of these plants.

In the 39-chromosome plants, a trivalent or univalent was invariably found in every cell (Table 2, Figs. 1 and 2). Trivalents were present in $43(56.58 \%)$ of the 76 cells studied. Such a high frequency of trivalents or univalents was not observed in the autotetraploid (Rao, unpublished). The average frequencies of quadrivalents, trivalents, bivalents and univalents per cell were 4.87, 0.57, 8.59 and 0.43 respectively. From the cytological studies it is apparent that the 39 -chromosome aneuploids arose by the union of gametes having 19 and 20 chromosomes.

In the 41-chromosome plants, as expected, a pentavalent was present in 49 $(44.55 \%)$ out of 110 cells studied and in the rest of them upto quadrivalent associations (Table 3, Figs. 3 and 4) were found. The mean frequencies of pentavalents, quadrivalents, trivalents, bivalents and univalents per cell were $0.54,5.55,0.03$, 8.16 and 0.53 respectively. It is thus apparent that the 41 -chromosome plants originated by mating of gametes with 20 and 21 chromosomes.

All the 40-chromosome progenies showed chromosome associations to the extent of quadrivalents only. These plants have probably arisen by fusion of gametes with 20 chromosomes.

Table 5. Chromosome numbers in the progenies of 39- and 41-chromosome plants of Job's tears

\begin{tabular}{ccccc}
\hline \multirow{2}{*}{$\begin{array}{c}\text { Chromosome } \\
\text { nos. (4n) }\end{array}$} & \multicolumn{2}{c}{ Progeny of $4 n=39$ plant } & \multicolumn{2}{c}{ Progeny of $4 n=41$ plant } \\
\cline { 2 - 5 } & Frequency & $\%$ & Frequency & $\%$ \\
\hline 39 & 5 & 16.13 & 4 & 10.26 \\
40 & 25 & 80.65 & 28 & 71.79 \\
41 & 1 & 3.22 & 7 & 17.95 \\
\hline
\end{tabular}

Although the possibility that the 39- and 41-chromosome plants could have arisen by the union of two aneuploid gametes ( 21 and 18, and 19 and 22 chromosomes respectively) does exist but such would be rare as the frequency of occurrence of aneuploid gametes itself was quite low that the chance of two such gametes coming together in mating would be further reduced. If such matings did occur, however, then some of the 39- and 41-chromosome plants could have shown multivalents of a much higher order than were actually recorded in this study. Similarly the 40-chromosome plants could also have come about by fusion of gametes with 21 and 19 , or 18 and 22 chromosomes. Nevertheless, since all the 40 -chromosome plants in the progeny contained multivalents of the order of only quadrivalents, it is obvious that such matings did not occur. Thus none of the 40-chromosome progenies examined were compensating aneuploids. The 40-chromosome plants, with typical autotetraploid behaviour, could, however, arise by mating of gametes with 19 and 21 chromosomes which are mutually compensating (deficient and excess for the same chromosome) but the probability of such matings would be extremely low.

Alternatively, it may be explained that zygotes resulting from mating between gametes having more than a single chromosome difference (Table 1) are inviable. 
Such zygotes might well be within the tolerable $39-41$ chromosome range, but still are inviable, due to quantitative reasons, if the same chromosome is in excess or deficient more than once. Similar situations were found also in maize (Shaver 1962), barley (Rommel 1961), red clover (Ellerström and Sjödin 1966) and ryegrass (Ahloowalia 1971).

\section{Fertility of aneuploids}

Both ovule and pollen fertility were determined from 14 plants in 39-chromosome class, 16 plants in 41-chromosome class and 22 plants in 40-chromosome class. It is likely that the different aneuploids could be deficient or excess for a different chromosome and that each one might exert its influence on fertility in a different way. Since it was not possible to identify one aneuploid from the other within the 39- or 41-chromosome class, the fertility data were collected together from plants, occurring in both $C_{1}$ and $C_{2}$ generations, in each of 39-, 40 and 41chromosome classes, and the mean fertility of these was shown in Table 4.

In pollen fertility, the aneuploids among themselves did not differ significantly, but in ovule fertility 41-chromosome plants were significantly higher than 39chromosome plants. Both pollen and ovule fertility in 40-chromosome plants were significantly higher than in aneuploids. Compared to pollen fertility, female fertility in all chromosome classes was low. This low female fertility was probably due to the method employed for determining the same, which includes both gametic inviability and embryo abortion. This probably also accounts for the low but positive coefficients of correlation between male and female fertility in individual plants of all chromosome classes (Table 4). The fact that aneuploids were signifcantly lower in fertility than euploids indicates that the state of their being aneuploid-with an imbalance in the chromosome number of the sporophyte-itself is probably responsible for it. Even though the aneuploid gametes were functional, seed set and pollen fertility were quite low indicating that all sterility in aneuploids and euploids might not be chromosomal but that much of it is genetic, as shown in many autopolyploids (Randolph 1935, Müntzing 1936, 1961, Rommel 1961, Shaver 1962, Ellerström and Sjödin 1966, Ahloowalia 1971).

Breeding behaviour of aneuploids

Open pollinated progenies of 39- and 41-chromosome plants were raised and the chromosome numbers in the progenies determined (Table 5). None of the progenies had chromosome numbers beyond the 39-41 range and most of the progenies (more than $70 \%$ ) showed $4 n=40$, the occurrence of which was as frequent as that in progenies of 40 -chromosome plants (Table 1). The average chromosome number of the progenies of 39-chromosome plants was 39.87 , and that of 41 -chromosome plants 40.08 . The corresponding figure for the progenies of 40 chromosome plants was 40.04 . This is suggestive that there is a rapid return to euploidy in the progenies of aneuploids.

\section{Summary}

In the progenies of autotetraploid $(4 n=40)$ Job's tears, aneuploids with 39 and 41 chromosomes occurred in some proportion. Non-recovery of aneuploids 
having less than 39 or more than 41 chromosomes was attributed to embryo abortion. The 39-chromosome plants regularly formed a trivalent or univalent in a cell and the 41-chromosome plants showed a pentavalent in a proportion of cells. Both aneuploid classes were less fertile than 40-chromosome plants. Much of the sterility in both aneuploids and euploids seemed to be genetic. Open pollinated progenies of 39 and 41-chromosome plants did not show chromosome numbers beyond the 39-41 range and a great majority of these exhibited an euploid number of 40 .

\section{Acknowledgements}

I am indebted to Professor J. Venkateswarlu under whose guidance this work was carried out.

\section{References}

Ahloowalia, B. S. 1971. Frequency, origin and survival of aneuploids in tetraploid rye grass. Genetica 42: 129-138.

Ellerström, S. and Sjödin, J. 1966. Frequency and vitality of aneuploids in a population of tetraploid red clover. Hereditas 55: 166-182.

Müntzing, A. 1936. The evolutionary significance of autopolyploidy. Hereditas 21:263-378.

- 1961. Genetic Research. Meijels Bokindustri, Stockholm, 1-345.

Randolph, L. F. 1935. Cytogenetics of tetraploid maize. J. Agr. Res. 50: 591-605.

Rommel, M. 1961. Aneuploidy, seed set and sterility in artificially induced autotetraploid Hordeum vulgare L. Can. J. Genet. Cytol. 3: 272-282.

Shaver, D. L. 1962. A study of aneuploidy in autotetraploid maize. Can. J. Genet. Cytol 4: 226-233.

Venkateswarlu, J. and Rao, P. N. 1974. Apomictic maternal diploids in tetraploid Job's tears. Theoret. Appl. Genet. 45: 274-276. 